\title{
Effects of Cupping Therapy on Lower Quarter Y-Balance Test Scores in Collegiate Baseball Players
}

ISSN: 2577-1914

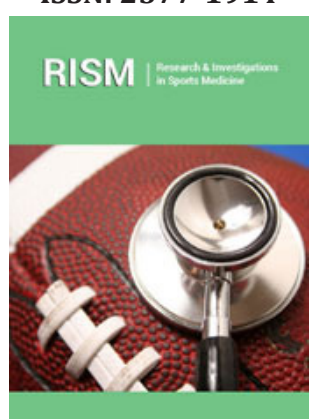

*Corresponding author: S Andrew C, University of Texas at Tyler, Texas, USA

Submission: 䤈 November 27, 2019

Published: 淟January 09, 2020

Volume 6 - Issue 1

How to cite this article: S Andrew C, Brandon W, Diana G, Kristen S. Effects of Cupping Therapy on Lower Quarter Y-Balance Test Scores in Collegiate Baseball Players. Res Inves Sports Med. 5(5). RISM.000627.2020.

DOI: 10.31031/RISM.2020.06.000627

Copyright@ S Andrew C, This article is distributed under the terms of the Creative Commons Attribution 4.0 International License, which permits unrestricted use and redistribution provided that the original author and source are credited.

\author{
S Andrew C*, Brandon W, Diana G and Kristen S \\ University of Texas at Tyler, USA \\ Grand Canyon University, USA \\ Christus Trinity Mother Frances, USA
}

\begin{abstract}
Cupping therapy is a therapeutic modality that has been suggested to improve blood flow, decrease pain, and increase function. Due to the scarcity of the literature in regard to best practices, parameters for prescribing and applying cupping therapy is absent in competitive athletic population. To ensure appropriate usage of cupping therapy, gaps in the literature on range of motion, strength, proprioception, power, speed and agility must be investigated. Therefore, the purpose of this study is to examine the effects of a single cupping therapy treatment to the gastrocnemius on Lower Quarter Y-Balance Test (YBT) scores in collegiate baseball players. Ten male (age $21.6 \pm 1.90$ ) collegiate baseball players participated in this study. Participants completed three attempts in the anterior, posterolateral, and posteromedial directions for both lower extremities per YBT procedures. Following baseline measurements, four plastic cups were applied to the gastrocnemius of the non-dominant lower extremity for 15-minutes. After treatment, follow up YBT scores were taken. Upon completion of data collection paired samples t-tests and oneway repeated measures ANOVAs were performed to determine significant differences in scores following cupping therapy. Significant improvements were found pre- and post-treatment in all three directions in the lower extremity receiving cupping therapy $(\mathrm{p}<0.05)$. When compared with the control extremity, significant differences were found in the anterior and posteromedial directions $(p<0.05)$. These results suggest that a single cupping therapy treatment to the gastrocnemius may improve anterior and posteromedial YBT scores in collegiate baseball players. Further studies are required to determine the effects of multiple cupping therapy treatments, as well as long term effects of cupping therapy on dynamic stability.
\end{abstract}

\section{Introduction}

Cupping therapy is an ancient therapeutic modality that used as early as 3300BC [1]. Previous studies have suggested that cupping therapy is an effective modality for reducing pain, increasing blood flow, increasing function, and increasing range of motion [2-4]. This increased range of motion has been attributed in theory to the increase in circulation that has been found with cupping therapy [5]. As high-level athletes in the United States use and promote cupping therapy, this treatment technique has grown in popularity. Even though cupping therapy has gained attention, there is still no consensus on the ideal parameters for applying cupping therapy to either professional or amateur athletes [1]. In the absence of an evidence driven best practices statement, an expert driven recommendation statement was created to serve as a resource for athletic trainers wishing to use cupping therapy in their clinical practice [6]. Although this clinical expert's statement was created, the authors did note that it was not intended to serve as an exhaustive document, but rather a resource to use while further research is conducted [6]. Despite research indicating that cupping therapy improves pain levels, blood flow, range of motion, and function, to the authors' knowledge there have not been any studies conducted on the effects of cupping therapy on lower extremity balance and strength.

The Lower Quarter Y-Balance Test (YBT) is a measure of lower extremity strength, neuromuscular control, and balance [7]. There have been some studies that suggest that lower scores on the YBT may be correlated with an increased risk of injury [8,9]. In previous studies, the YBT has been shown to be a valid method of assessing lower extremity strength, 
neuromuscular control, and balance in baseball pitchers $[7,10]$. The YBT-anterior reach has also been suggested as a useful tool to help identified decreased stride length in baseball pitchers [7]. This decreased stride length has been associated with increased stress to the shoulder and elbow and pitching mechanics defects [7]. This combination of factors may lead to an increased risk of upper extremity injury [7]. Being able to improve the performance characteristics assessed by the YBT, may help decrease the risk of musculoskeletal injury $[7,10]$. Thus, the aim of this study is to determine the effects of a single 15-minute cupping therapy treatment to the calf musculature on YBT scores in collegiate baseball players.

\section{Methods}

\section{Participants}

Ten male (age $21.6 \pm 1.90$ years; height $175.61 \pm 21.41 \mathrm{~cm}$; weight $80.33 \pm 8.07 \mathrm{~kg}$ ) collegiate baseball players participated in this study. All subjects were members of an NCAA Division II baseball team. All subjects were screened for a history of significant lower extremity injury, which was defined as a lower extremity injury that resulted in any lost time from practice or competition. Subjects were educated on the risks and benefits of the study and provided informed consent prior to the beginning of data collection.

\section{Data collection}

In this study, YBT scores were taken in the anterior, posteromedial, and posterolateral directions for both legs. Subjects completed three trials in each direction for both legs. Per YBT protocol, the highest score in each direction was used for data analysis [7]. Following completion of baseline testing, the subjects were then placed in a prone position. The left gastrocnemius muscle was prepared for treatment with coconut oil, and then four plastic pneumatic cups were then applied for 15 minutes. During this time, the right gastrocnemius received no treatment in order to serve as a control. Upon completion of cupping therapy treatment, the cups were removed, and the skin was cleaned with a terry cloth towel. Then, the subjects performed a second YBT protocol for both legs. At this time, the subjects were dismissed once they confirmed that they had no further questions and had not suffered any adverse responses to cupping therapy treatment.

\section{Statistical analysis}

After data collection was completed, data was compiled and analyzed for statistical significance. Paired samples t-tests were run for all three directions to assess the difference pre- and postintervention. Between group measures using one-way repeated ANOVAs were performed to assess differences between all three directions of the YBT post cupping therapy. All statistical tests were performed using SPSS V26 (IBM, Armonk, NY).

\section{Result}

Means and standard deviations for YBT scores is presented in Table 1. In all three directions, reach distance increased at a significant rate (Anterior $=65.45 \pm 4.44$ to $70.15 \pm 5.85$, Posteromedial $=105.70 \pm 4.92$ to $110.45 \pm 5.35$, Posterolateral $=$ $110.25 \pm 7.46$ to $114.6 \pm 6.38, p<0.05$ ). In addition to significant improvements when comparing pre- and post-intervention reach scores, subjects experienced significantly increased reach scores in the anterior and posteromedial directions when compared to their control leg (Anterior Treatment $=4.5 \pm 3.89$, Anterior Control $=$ $-1.15 \pm 4.57$, Posteromedial Treatment $=4.75 \pm 3.65$, Posteromedial Control $=0.00 \pm 3.86, \mathrm{p}<0.05$ ) (Table 2).

Table 1: Mean \pm SD scores for Lower Quarter Y-Balance Test scores for treated limb in $\mathrm{cm}$.

\begin{tabular}{|c|c|c|}
\hline & YBT Scores Before and After Cupping Therapy & Post-Treatment \\
\hline Direction & Pre-Treatment & $70.15 \pm 5.85^{*}$ \\
\hline Anterior & $65.65 \pm 4.44$ & $110.45 \pm 5.35^{*}$ \\
\hline Posteromedial & $105.70 \pm 4.92$ & $114.6 \pm 6.38^{*}$ \\
\hline Posterolateral & $110.25 \pm 7.46$ & \\
\hline
\end{tabular}

* Reach was significantly different that previous condition.

Table 2: Mean \pm SD score differences for treatment and control limbs in $\mathrm{cm}$.

\begin{tabular}{|c|c|c|}
\hline \multicolumn{2}{|c|}{ YBT Score Differences in Cupping Compared to Control } \\
\hline Direction & Treatment & Control \\
\hline Anterior & $4.50 \pm 3.89^{*}$ & $-1.15 \pm 4.57$ \\
\hline Posteromedial & $4.75 \pm 3.65^{*}$ & $0.00 \pm 3.86$ \\
\hline Posterolateral & $4.35 \pm 4.36$ & $1.70 \pm 4.85$ \\
\hline
\end{tabular}

*Reach was significantly different from compare condition.

\section{Discussion}

The purpose of this study was to determine if a single dry cupping therapy treatment would have an influence on YBT scores. Lower composite scores on the YBT have been correlated with increased risk of injury [7]. While these studies cannot definitively conclude that an increased score on the YBT would decrease the risk of injury, improvements would address a potential risk factor. Results from the current study suggest that a 15-minute 
cupping therapy treatment to the medial and lateral heads of the gastrocnemius result in significant improvements on the YBT in the anterior and posteromedial directions when compared to a control group. This increase in YBT scores may be indicative of an acute increase in balance and neuromuscular control. The authors theorized these increases may be the result of a decrease in muscle stiffness and increase in muscle flexibility.

The specific mechanisms for cupping therapy decreasing muscle stiffness are not well understood. It has been suggested that decreases in pain and increases in myofascial space may activate neuromodulatory effects, such as the "gate control theory", resulting in an overall decreased muscle stiffness [4]. Another theory is that the increase in blood flow caused by cupping therapy plays a role in increasing muscle flexibility [5]. However, there do not appear to be many published studies examining the effects cupping therapy may have on muscle stiffness and range of motion.

A possible limitation of this study was the relatively small sample size. A follow-up study involving a large subject population may allow for a more conclusive statement to be made on the effects of cupping therapy on YBT scores in baseball players. Another potential limitation was that the authors were unable to determine whether the increased scores were due to improvements in lower extremity balance, strength, range of motion, or neuromuscular control. Further research should be done using validated patient and clinician-based outcomes measures to better determine the effects of cupping therapy has on muscular performance.

To the authors' knowledge, this is the first study conducted to assess the effects of cupping therapy on lower extremity balance, strength, range of motion, or neuromuscular control in an athletic population. Future research should look further into the effects of cupping therapy on other measures of muscular performance. Provided these studies use similar methodology, a more conclusive systematic review may be able to be performed on the use of cupping therapy with athletic populations.

\section{References}

1. Bridgett R, Klose P, Duffield R, Mydock S, Lauche R (2018) Effects of cupping therapy in amateur and professional athletes: Systematic review of randomized controlled trial. J Altern Complement Med 24(3): 208-219.

2. Arce-Esquivel A, Warner B, Gallegos D, Cage S (2017) Effect of dry cupping on vascular function among young individuals. International Journal of Health Sciences 5(3): 10-15.

3. Cao H, Li X, Yan X, Wang N, Bensoussan A, et al. (2014) Cupping therapy for acute and chronic pain management: A systematic review. Journal of Traditional Chinese Medical Sciences 1(1): 49-61.

4. Lowe DT (2017) Cupping therapy: An analysis of the effects of suction on the skin and possible influence on human health. Complement Ther Clin Pract 29: 162-168.

5. Markowski A, Sanford S, Pikowski J, Fauvell D, Cimino D, et al. (2014) A pilot study analyzing the effects of Chinese cupping as an adjunct treatment for patients with subacute low back pain on relieving pain, improving range of motion, and improving function. J Altern Complement Med 20(2): 113-117.

6. Cage SA, Gallegos DM, Coulbome B, Warner BJ (2019) Clinical experts' statement: The definition, prescription, and application of dry cupping therapy. Clinical Practice in Athletic Training 2(2): 4-11.

7. Culliver A, Garrison JC, Creed KM, Conway JE, Goto S, et al. (2019) Correlation among Y-Balance Test-Lower Quarter composite scores, hip musculoskeletal characteristics, and pitching kinematics in NCAA Division I baseball pitchers. J Sport Rehabil 289(5): 432-437.

8. Hartley EM, Hoch MC, Bling MC (2018) Y-Balance Test performance and BMI are associated with ankle sprain injury in collegiate male athletes. J Sci Med Sport 21(7): 676-680.

9. Smith CA, Chimera NJ, Warren M (2015) Association of Y-Balance Test reach asymmetry and injury in Division I athletes Med Sci Sports Exerc 47(1): 136-141.

10. Butler RJ, Bullock G, Arnold T, Plisky P, Queen R (2016) Competition-level difference on lower quarter Y-Balance Test in baseball players. J Athl Train 51(12): 997-1002. 